\title{
A chromosomal passenger complex protein signature model predicts poor prognosis for non-small-cell lung cancer
}

This article was published in the following Dove Press journal:

OncoTargets and Therapy

7 April 2015

Number of times this article has been viewed

\author{
Rong Xial,* \\ Sunxiao Chen ${ }^{2, *}$ \\ Yan Chen ${ }^{3}$ \\ Weiwei Zhang ${ }^{3}$ \\ Rongrong $\mathrm{Zhu}^{3}$ \\ Anmei Deng ${ }^{3}$ \\ 'Department of Transfusion, \\ Huashan Hospital, Fudan University, \\ 2Department of Dermatology, \\ Changzheng Hospital, Second Military \\ Medical University, ${ }^{3}$ Department \\ of Laboratory Diagnosis, Changhai \\ Hospital, Second Military Medical \\ University, Shanghai, People's Republic \\ of China \\ *These authors contributed equally \\ to this work
}

Correspondence: Anmei Deng Department of Laboratory Diagnosis, Changhai Hospital, Second Military Medical University, 168 Changhai Road, Shanghai 200433, People's Republic of China

Tel +862131162061

Fax +86 2I 3116206I

Email anmeideng@aliyun.com
Aim: The chromosomal passenger complex (CPC) acts as a key modulator for mitosis and cell cytokinesis. High levels of CPC proteins are frequently observed in multiple cancers and are correlated with more progressive malignant behaviors. The aim of the study was to evaluate whether CPC components or their combinations could be used to assess the clinical risk of patients with non-small-cell lung cancer (NSCLC).

Methods: The expression levels of four CPC proteins - aurora B kinase (AURKB), borealin, inner centromere protein (INCENP), and survivin - were evaluated using immunohistochemistry in an independent cohort of NSCLC specimens. A molecular predictor model was developed based on the combination of the four CPC proteins.

Results: All the CPC components were overexpressed in NSCLC tumors compared with their paired adjacent normal lung tissues. Survivin overexpression was significantly correlated with late tumor stage $(P=0.0166)$. High expressions of AURKB, INCENP, and survivin, but not borealin, were associated with shorter survival in patients with NSCLC. The constructed 4-CPC-gene model divided the cohort into two different subgroups with significantly different prognoses (hazard ratio, $\mathrm{HR}=2.8915$ [95\% confidence interval, $\mathrm{CI}$ : $1.5187-5.5052$ ]; $P=0.0013$ ) and was retained as an independent prognostic factor in multivariate analysis $(\mathrm{HR}=2.4398$ [95\% CI: 1.2631-4.7127], $P=0.0082)$. Moreover, the 4-CPC-gene model demonstrated a higher predictive ability for overall survival than each individual CPC biomarker.

Conclusion: Taken together, our study suggests that a molecular prognostic model based on simultaneous detection of CPC components could serve as a complement to current clinical risk stratification approaches for patients with NSCLC.

Keywords: non-small-cell lung cancer, chromosomal passenger complex, AURKB, survivin, borealin, INCENP

\section{Introduction}

Lung cancer is the leading cause of cancer-related deaths worldwide, including the People's Republic of China. Non-small-cell lung cancer (NSCLC) accounts for approximately $85 \%$ of all the lung cancer cases. The intrinsic heterogeneity of NSCLC makes it necessary to find novel single or combined biomarkers to accurately identify patient subgroups with different clinical outcomes.

The chromosomal passenger complex (CPC), composed of aurora B kinase (AURKB), borealin, inner centromere protein (INCENP), and survivin, acts a critical mitotic regulator to accurately control the events involved in the mitotic cell cycle, which are known to play crucial roles in the expansion of tumor cells. ${ }^{1,2}$ Interestingly, increased levels of some of these components such as AURKB and survivin have been observed in a spectrum of cancers, including NSCLC, and predict early tumor 
recurrence and poor prognosis., ${ }^{3,4}$ But the clinical significance of two other CPC proteins - borealin and INCENP in NSCLC has not been investigated in previous studies. Furthermore, all the previous studies focused on the clinical significance of a single CPC component in cancers. Accumulating evidence indicates that combining biomarkers belonging to the same cancer hallmarks might better reflect tumor biology and aggressiveness and thereby could increase their clinical discriminative and prognostic value synergistically. ${ }^{5}$ Therefore, in this study, we investigated the expression of four CPC components in NSCLC; the clinicopathological and prognostic values of individual biomarkers and their combination with patients' survival were also evaluated.

\section{Materials and methods Study cohort and samples}

Archived paraffin-embedded tissues were derived from an independent cohort of patients with NSCLC undergoing curative resection between July 2004 and August 2008. Treatments followed the National Comprehensive Cancer Network non-small-cell lung cancer Clinical Practice Guidelines (Chinese version). All the relevant clinical and survival data of patients with NSCLC were available. Patients who died of non-cancer-related causes or died within 30 days after surgery were excluded. Finally, 104 NSCLC specimens were selected in the study. The median follow-up period for surviving patients was 40 months (ranging from 8 months to 89 months). This study was approved by our local ethics committees in accordance with the proposals of International Ethical Guideline for Biomedical Research (CIOMS). The clinical characteristics across the cohorts are summarized in Table 1.

\section{Assessment of CPC biomarker expression by immunohistochemistry}

Formalin-fixed, paraffin-embedded 5- $\mu$ m-thick sections were subjected to immunohistochemical staining with the avidinbiotin-peroxidase complex system. After deparaffinization, endogenous peroxidases were quenched in $3 \% \mathrm{H}_{2} \mathrm{O}_{2}$ for 20 minutes. Antigen retrieval was performed by heating in $10 \mathrm{mM}$ citrate buffer $\left(\mathrm{pH} 6.0\right.$ ) at $95^{\circ} \mathrm{C}$ for 60 minutes. Sections were then incubated at $4^{\circ} \mathrm{C}$ overnight with mouse or rabbit antibodies against human AURKB (Santa Cruz, 1:150), borealin (Santa Cruz, 1:100), INCENP (Santa Cruz, 1:150), and survivin (Santa Cruz, 1:100). Detection was performed with the avidin-biotin-peroxidase complex system (Dako Envision) using diaminobenzidine as the chromogen. Slides were then counterstained with hematoxylin.
Table I Clinical characteristics across the cohorts

\begin{tabular}{lll}
\hline & Number of cases & $\%$ \\
\hline Age (years) & 72 & \\
$\quad<60$ & 32 & 69.2 \\
$\geq 60$ & & 30.8 \\
Sex & 78 & 75 \\
$\quad$ Male & 26 & 25 \\
$\quad$ Female & & \\
Tumor type & 52 & 50 \\
$\quad$ Adenocarcinoma & 52 & 50 \\
Squamous cell carcinomas & & \\
Grade & 15 & 14.4 \\
I & 51 & 49.1 \\
2 & 38 & 36.5 \\
3 & & \\
Tumor size & 86 & 82.7 \\
I-2 & 18 & 17.3 \\
$3-4$ & & \\
Lymph node metastasis & 48 & 46.2 \\
Positive & 56 & 53.8 \\
Negative & & 68.3 \\
Stage & 71 & 31.7 \\
I-2 & 33 & \\
$3-4$ & &
\end{tabular}

Nonspecific mouse or rabbit immunoglobulin G was used as the negative control.

\section{Evaluation of immunohistochemical results}

The results of CPC protein immunostaining were scored by multiplying the intensity score $(0=$ negative, $1=$ weak, $2=$ moderate, and $3=$ strong) and the percentage of the total cell population (proportion score: $0 \%=0,1 \%-10 \%=1$, $11 \%-50 \%=2,51 \%-80 \%=3,81 \%-100 \%=4)$. On the basis of the distributions of the expression scores, the median scores were used as cutoff values to divide patients into high- and low-expression subgroups for each of the individual markers, as described previously. ${ }^{6}$

\section{Construction of 4-CPC-gene prognostic model}

The weighted overall survival predictive model using multiple biomarkers was constructed as described previously., ${ }^{7,8}$ Briefly, the association between individual CPC proteins and overall survival of patients with NSCLC was evaluated by univariate Cox proportional-hazards regression analysis. Then, a predictive model of combined biomarkers was constructed by summing the expression level of each biomarker multiplied by its regression coefficient in univariate Cox analysis. Patients with NSCLC were classified into high- and 
low-expression subgroups using the 50th percentile (median) cutoff of the combined values in our constructed model.

\section{Statistics}

The relationship between the single biomarker or combined model and the characteristics was analyzed using the $\chi^{2}$ test. Correlations between the expression levels of single biomarkers were assessed by the Spearman rank correlation coefficients. The Kaplan-Meier method was used for survival analysis and results were evaluated by the log-rank test. Univariate and multivariate analyses were performed using a Cox proportional-hazards model. The receiver operating characteristic curves were used to measure the predictive performance for the prognostic factors. All the analyses were performed using the MedCalc 11.0 software. Differences were considered statistically significant at $P<0.05$.

\section{Results}

The four CPC proteins were predominantly expressed in the cell nuclei of both normal lung and paired adjacent NSCLC tissues (Figure 1). The staining intensity and density of all these CPC proteins in NSCLC tissues were significantly higher than those in paired adjacent normal lung tissues. Spearman's rank correlation analysis showed that the expression of AURKB was significantly associated with survivin expression ( $r=0.2500, P=0.0105)$, while no significant correlation existed among the other biomarkers.

We further assessed the association of CPC components with clinical and pathological parameters of the patients with NSCLC, including age, tumor type, differentiation, grade, and stage. The factor showing significant correlation was survivin, with tumor stage; high survivin expression cases were more frequently observed in late-stage tumors than in

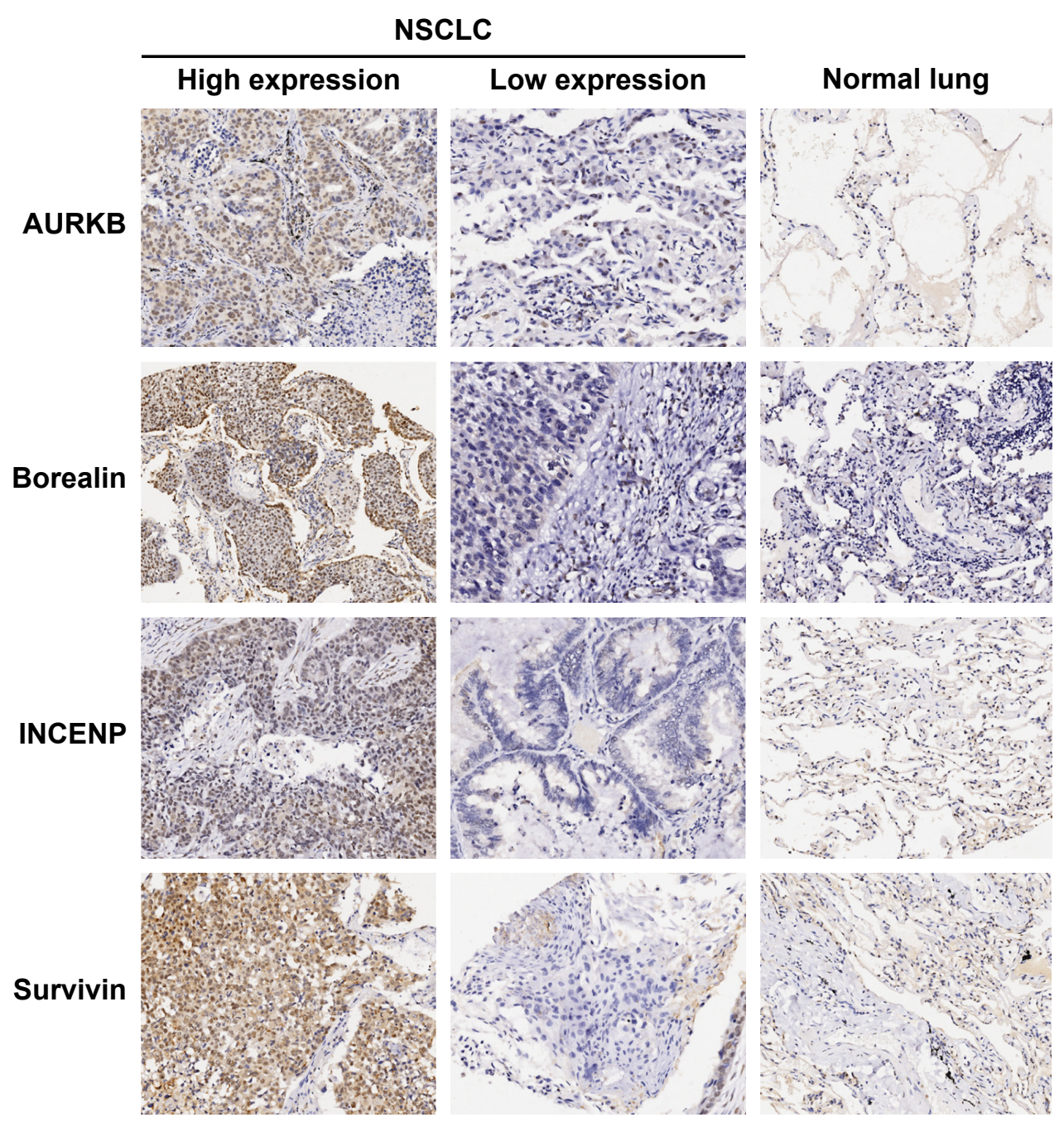

Figure I Representative images of immunostaining of AURKB, borealin, INCENP, and survivin in NSCLC and normal lung tissues ( $\times 100$ magnification). Abbreviations: AURKB, aurora B kinase; INCENP, inner centromere protein; NSCLC, non-small-cell lung cancer. 
early-stage tumors $(69.7 \%$ vs $42.2 \%, P=0.0166)$. There was no other significant correlation observed between the expression levels of CPCs and clinical parameters of NSCLC.

Then we used the Kaplan-Meier curves and the univariate Cox proportional-hazards model to identify the association between the CPCs and patients' overall survival. As seen in Figure 2A-D and Table 2, high levels of AURKB, INCENP, and survivin were significantly associated with shorter survival in patients with NSCLC $(P=0.0414, P=0.0355$, and $P=0.0053$, respectively). Meanwhile, the high expression status of borealin was also correlated with poor prognosis in NSCLC, but it only had a marginal significance $(P=0.0728)$. We also found that INCENP and survivin were independent prognostic factors for patients with NSCLC in Cox regression models. For survivin expression levels significantly correlated with tumor stage, we further analyzed the correlation between survivin and survival in the same stage and found that although high survivin level is associated with poor prognosis in both early and late stages, a statistically significant difference was not observed.

Next, we built up a 4-CPC-gene signature model by summing the expression level of each biomarker (high expression $=1$, low expression $=0$ ) multiplied by its coefficient value from univariate Cox analysis. Then, the 104 NSCLC cases were ranked and divided into high- and lowrisk groups using the 50th percentile (median) cutoff of the combined score value. Our Kaplan-Meier test demonstrated that the high-risk group classified by the combined model had a shorter overall survival than the low-risk group (Table 2 and Figure 2E). In the Cox regression model, the 4-CPC-gene model was retained as an independent prognostic factor. The receiver operating characteristic analysis showed that the

A

Status

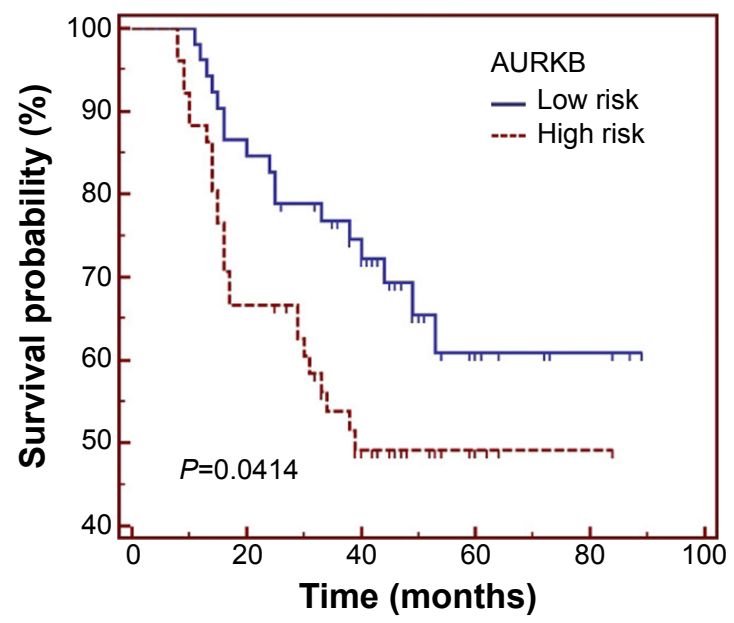

predictive power of the combined 4-CPC-gene prognostic model was higher than that of the individual CPC proteins (Figure 3).

\section{Discussion}

The clinical and prognostic significance of single CPC components has been investigated in multiple cancers, including lung cancers. ${ }^{9,10}$ Clinical and prognostic implications of survivin in lung cancers have been investigated in multiple studies. Several meta-analysis publications ${ }^{11,12}$ support the result that upregulated survivin predicts poor prognosis in patients with NSCLC (combined hazard ratio [HR] ranged from 1.88 to 1.95 ). Takeshita et $\mathrm{al}^{13}$ showed that overexpression of AURKB is significantly correlated with aneuploidy and poor outcomes in NSCLC. The expression levels of borealin and INCENP and their clinicopathological and prognostic significance have not been investigated in previous studies. To our knowledge, this study is the first to report the prognostic value of the combination of CPC biomarkers in a cohort of cancer patients.

In this study, we immunohistochemically investigated the expression of CPC proteins in NSCLC tissues. Consistent with previous findings, we validated that survivin and AURKB were associated with more malignant phenotypes and that they were independent prognostic factors for NSCLC. We also found that the other two CPC proteins borealin and INCENP were upregulated in NSCLC and were associated with tumor progression and survival, but only at a marginal significance for borealin.

Among these markers, we found that AURKB overexpression was closely associated with that of survivin in NSCLC. Similar positive correlation between survivin and AURKB has also been reported in head and neck cancer. ${ }^{14}$ In

B

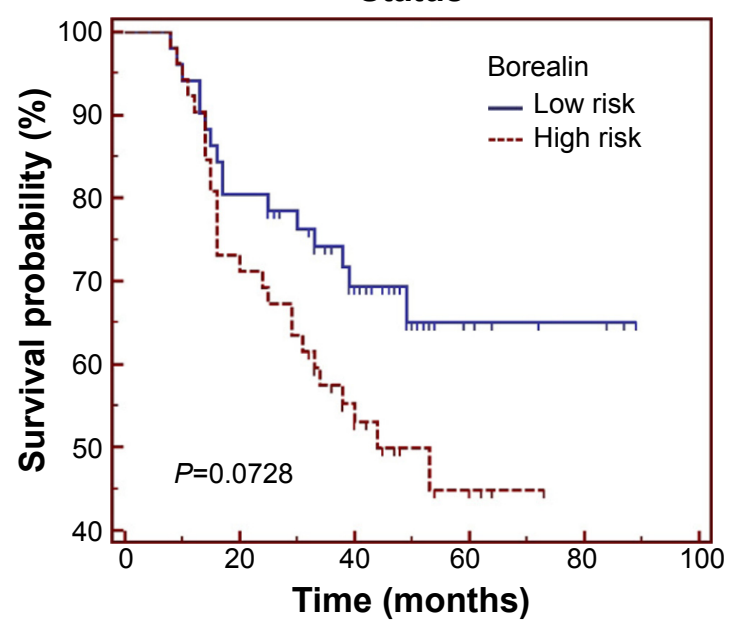

Figure 2 (Continued) 

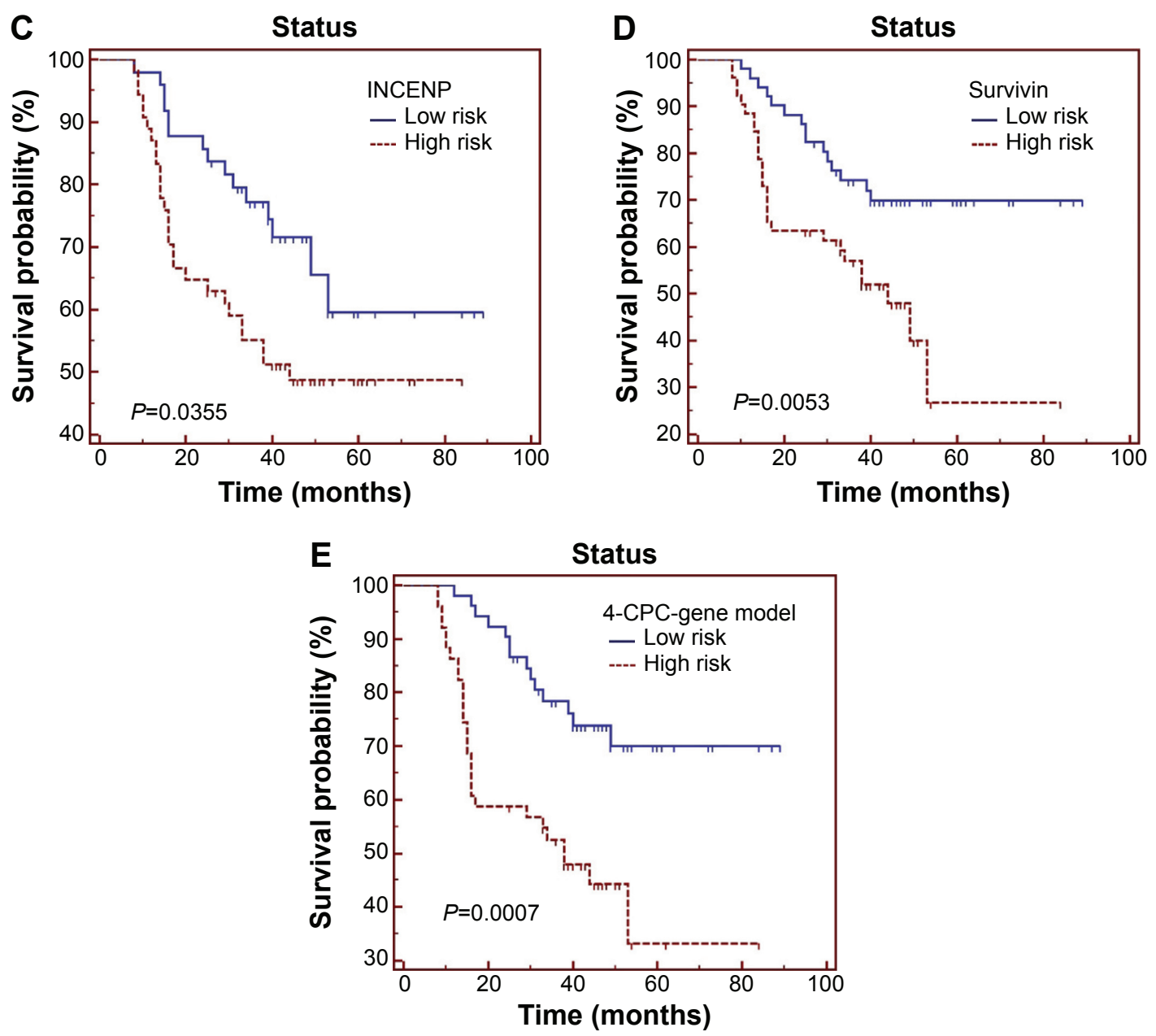

Figure 2 Overall survival of 104 patients with NSCLC in relation to (A) AURKB, (B) borealin, (C) INCENP, and (D) survivin, individually, and (E) the 4-CPC-gene prognostic model. Data were evaluated using the Kaplan-Meier survival test. Blue bars represent low-risk subgroup defined by 4-CPC-gene model, and red bars represent high risk subgroup. Abbreviations: AURKB, aurora B kinase; CPC, chromosomal passenger complex; INCENP, inner centromere protein; NSCLC, non-small-cell lung cancer.

an in vitro study, Chang et al ${ }^{15}$ found that borealin interacts with survivin but not with AURKB in gastric cancer cells. AURKB was demonstrated to be coexpressed in gastric cancer cells and to be colocalized with survivin during mitosis. Furthermore, knockdown of survivin could promote the degradation of AURKB, and vice versa. ${ }^{15}$ However, the other $\mathrm{CPC}$ components have no significant correlation with each other. The above findings suggest that these proteins might individually contribute to aberrant CPC pathways in cancer.
Therefore, it is reasonable to speculate that considering the expression levels of these markers together would represent the degree of CPC dysfunction of cancer cells better.

In this study, we demonstrated that a 4-CPC-gene model was a strong predictor for shorter survival in patients with NSCLC, with a higher HR nearly up to 3.0, which is superior to the HR for the established individual prognostic predictor survivin and other single CPC biomarkers. Our analyses implicated that tumors with an increasing

Table 2 Univariate and multivariate analyses of CPC factors associated with the overall survival

\begin{tabular}{|c|c|c|c|c|c|c|c|c|}
\hline \multirow[t]{3}{*}{ Variables } & \multicolumn{4}{|c|}{ Univariate analysis } & \multicolumn{4}{|c|}{ Multivariate analysis } \\
\hline & \multirow[t]{2}{*}{ Significance } & \multirow[t]{2}{*}{ HR } & \multicolumn{2}{|c|}{ 95\% Cl for $\mathrm{HR}$} & \multirow[t]{2}{*}{ Significance } & \multirow[t]{2}{*}{ HR } & \multicolumn{2}{|c|}{$95 \% \mathrm{Cl}$ for $\mathrm{HR}$} \\
\hline & & & Lower & Upper & & & Lower & Upper \\
\hline AURKB & 0.0465 & $|.874|$ & 1.0130 & 3.4672 & 0.0546 & 1.8573 & 0.9912 & 3.4804 \\
\hline Borealin & 0.0789 & I.7486 & 0.9404 & 3.2515 & 0.1950 & 1.5187 & 0.8098 & 2.8483 \\
\hline INCENP & 0.0404 & 1.9362 & 1.0325 & 3.6310 & 0.0211 & 2.1346 & 1.1243 & 4.0526 \\
\hline Survivin & 0.0072 & 2.4002 & 1.2709 & 4.5329 & 0.0345 & 2.0602 & 1.0576 & 4.0135 \\
\hline $\begin{array}{l}\text { 4-CPC-gene prognostic } \\
\text { model }\end{array}$ & 0.0013 & 2.8915 & 1.5187 & 5.5052 & 0.0082 & 2.4398 & $|.263|$ & 4.7127 \\
\hline
\end{tabular}

Abbreviations: AURKB, aurora B kinase; Cl, confidence interval; CPC, chromosomal passenger complex; INCENP, inner centromere protein; HR, hazard ratio. 


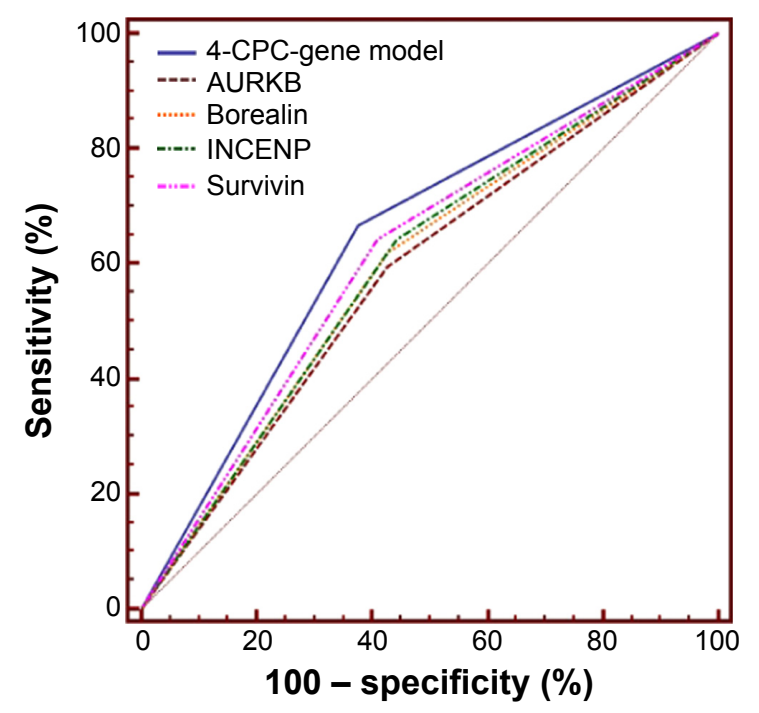

Figure 3 ROC analysis showed that the predictive power of the 4-CPC-gene prognostic model was higher than that of each CPC biomarker individually. Abbreviations: $A \cup R K B$, aurora $B$ kinase; $C P C$, chromosomal passenger complex; INCENP, inner centromere protein; ROC, receiver operating characteristic.

number of biomarkers may better reflect the underlying CPC pathway aberrations and thereby could identify patients with more aggressive phenotypes and clinically unfavorable prognosis. Currently, several CPC inhibitors are under development for cancer treatments. Therefore, we also proposed that CPC components could be potential targets for a portion of patients with NSCLC with relatively poor prognosis.

In conclusion, in this study, we showed that most of the CPC proteins were correlated with poor clinical outcome for NSCLC. Furthermore, a molecular prognostic model based on the combined four CPC proteins demonstrated a superior predictive ability relative to the individual biomarkers. The proposed biomarker combination deserves further investigation in use under clinical settings.

\section{Acknowledgments}

This study was supported by grants from the National Basic Research Program of China (973 Program) (2013CB531606), the National Science Foundation of China (81471605, 81472770, 81272280), and the Shanghai Municipal Commission for Science and Technology (11JC1410902).

OncoTargets and Therapy

\section{Publish your work in this journal}

OncoTargets and Therapy is an international, peer-reviewed, open access journal focusing on the pathological basis of all cancers, potential targets for therapy and treatment protocols employed to improve the management of cancer patients. The journal also focuses on the impact of management programs and new therapeutic agents and protocols on

\section{Disclosure}

The authors report no conflicts of interest in this work.

\section{References}

1. Carmena M, Wheelock M, Funabiki H, Earnshaw WC. The chromosomal passenger complex (CPC): from easy rider to the godfather of mitosis. Nat Rev Mol Cell Biol. 2012;13(12):789-803.

2. van der Waal MS, Hengeveld RC, van der Horst A, Lens SM. Cell division control by the chromosomal passenger complex. Exp Cell Res. 2012;318(12):1407-1420.

3. Portella G, Passaro C, Chieffi P. Aurora B: a new prognostic marker and therapeutic target in cancer. Curr Med Chem. 2011;18(4):482-496.

4. Athanasoula K, Gogas H, Polonifi K, Vaiopoulos AG, Polyzos A, Mantzourani M. Survivin beyond physiology: orchestration of multistep carcinogenesis and therapeutic potentials. Cancer Lett. 2014;347(2):175-182.

5. Goossens-Beumer IJ, Zeestraten EC, Benard A, et al. Clinical prognostic value of combined analysis of Aldh1, Survivin, and EpCAM expression in colorectal cancer. Br J Cancer. 2014;110(12):2935-2944.

6. Li XJ, Ong CK, Cao Y, et al. Serglycin is a theranostic target in nasopharyngeal carcinoma that promotes metastasis. Cancer Res. 2011;71(8):3162-3172.

7. Peng ZM, Yu W, Xie Y, et al. A four actin-binding protein signature model for poor prognosis of patients with esophageal squamous cell carcinoma. Int J Clin Exp Pathol. 2014;7(9):5950-5959.

8. Cao HH, Zheng CP, Wang SH, et al. A molecular prognostic model predicts esophageal squamous cell carcinoma prognosis. PLoS One. 2014;9(8):e106007.

9. Yoshida A, Zokumasu K, Wano Y, et al. Marked upregulation of Survivin and Aurora-B kinase is associated with disease progression in the myelodysplastic syndromes. Haematologica. 2012;97(9):1372-1379.

10. Erpolat OP, Gocun PU, Akmansu M, Karakus E, Akyol G. High expression of nuclear survivin and Aurora B predicts poor overall survival in patients with head and neck squamous cell cancer. Strahlenther Onkol. 2012;188(3):248-254.

11. Huang LN, Wang DS, Chen YQ, et al. Expression of survivin and patients survival in non-small cell lung cancer: a meta-analysis of the published studies. Mol Biol Rep. 2013;40(2):917-924.

12. Fan J, Wang L, Jiang GN, He WX, Ding JA. The role of survivin on overall survival of non-small cell lung cancer, a meta-analysis of published literatures. Lung Cancer. 2008;61(1):91-96.

13. Takeshita M, Koga T, Takayama K, et al. Aurora-B overexpression is correlated with aneuploidy and poor prognosis in non-small cell lung cancer. Lung Cancer. 2013;80(1):85-90.

14. Qi G, Kudo Y, Ando T, et al. Nuclear Survivin expression is correlated with malignant behaviors of head and neck cancer together with Aurora-B. Oral Oncol. 2010;46(4):263-270.

15. Chang JL, Chen TH, Wang CF, et al. Borealin/Dasra B is a cell cycleregulated chromosomal passenger protein and its nuclear accumulation is linked to poor prognosis for human gastric cancer. Exp Cell Res. 2006;312(7):962-973.

\section{Dovepress}

patient perspectives such as quality of life, adherence and satisfaction. The manuscript management system is completely online and includes a very quick and fair peer-review system, which is all easy to use. Visit http://www.dovepress.com/testimonials.php to read real quotes from published authors. 